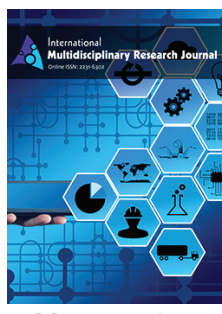

ISSN: $2231-6302$

Received: February 15,2020

Accepted: March 31,2020

Published: April 11, 2020

*Corresponding Author:

Rabab Farhan Abbas

Email: 70154@uotechnology.

edu.iq

\title{
Review on some methods used in image restoration
}

\author{
Rabab Farhan Abbas \\ Specification of Computer science Working place: Department of Production Engineering and metallurgy, \\ University of Technology Baghdad, Iraq
}

\begin{abstract}
The restoration image is manner of mending the inventive image by eradicating noise and fuzziness from image. Image fuzziness is troublesome to shun in several things similar shooting, to confiscate motion blur caused by camera stillness, measuring device imaging to eradicate the outcome of image scheme retort, etc. The aim of image restoration is guesstimate the innovative image from surveillance image despoiled by haziness and preservative noise as much as promising. Altered image restoration techniques have urbanized by many researches. In this review I will discuss different images restoration methods.
\end{abstract}

KEYWORDS: Image restoration, mean filter, median filter

\section{INTRODUCTION}

Digital images are electronic snapshots of a scene, that composed of generally image components in an exceedingly grid formation called pixels, every pixel holds a price that is quintal that represents the tone at a particular purpose. Images are gotten in capacities starting from commonplace shooting to urology, far-flung sensing, microscopy, health imaging etc. [1].

Image restoration states to the retrieval of an unidentified true image from its degraded measurement. The degradation could occur throughout image formation, transmission, and storage; and it's going to be in an exceedingly variety of forms, as well as additive noise [2], area invariant or variant blur, aliasing, and compression whole. With the progresses in imaging, computing, and communication technologies over the historical periods, image restoration has evolved into a field at the intersection of image process, pc vision, and machine imaging. Its derivatives embrace image denoising (also referred to as noise removal/ reduction), image deblurring/deconvolution (including optical/motion deblurring), image in painting (also known as image completion), image interpolation (including super resolution and color demosaicking), image reconstruction (including computerized axial tomography and compressed sensing), and image deblocking/deranging (also mentioned as compression whole removal). Apparently image restoration techniques became an elementary tool to support low-level vision tasks arising from numerous scientific and engineering fields [3]. The degradation method is viewed with the subsequent system. The degraded operate is low pass filter [4].

As per recognized in (figure 1 ) the innovative contribution is a two-dimensional image $\mathrm{f}(\mathrm{x}, \mathrm{y})$. This spitting image is functioned on the scheme $h(x, y)$ and later the accumulation of noise $\mathrm{n}(\mathrm{x}, \mathrm{y})$. One be able to get the despoiled image $\mathrm{g}(\mathrm{x}, \mathrm{y})$ [4]. Cardinal image restoration can be envisioned by way of method in which crack is gotten a guesstimate to $f(x, y)$. The blurred image can be labelled with the next equation [5].

$$
g(x, y)=h(x, y) * f(x, y)+n(x, y)
$$

\section{IMAGE RESTORATION REQUESTS}

The requests within the turf of image restoration are:

- The request of ordinal image restoration within the manufacturing communal is the space of planetary imaging. Otherworldly observations of the world and therefore the spheres were tainted by gesture blur as a results of unhurried camera shutter haste relative to hurried ballistic capsule motion. The planetary imaging poverty drawback is commonly categorized by Poisson noise, Gaussian noise etc. [6]

- In the creation of medical imaging, image restoration takes vie an awfully vital character. Restoration takes cast-off for mammograms, clarifying of Poisson spread film scrap noise in chest X-rays and numeral angiographic movies, and for the removal of additive noise in quality imaging [7]. 
- Alternative spirited request of restoration procedure is to revive elderly and declined movies. The depiction restoration is commonly related to numerical methods are accustomed eradicate cuts and dirt from recent flicks and additionally two-color in white and black films. Nearby important improve the world of restoration of image sequences and well described in literature [6].

- The cumulative space of request for cardinal image restoration is that inside the arena of image and video furtive writing. Such as methods are developed to enhance secret script force, and scale spinal the bit duties of coded movies. A lot of takes accomplishes to ripen ways that of returning coded movies as a post dispensation stage to be done once decompression [7].

- Numerical image retrieval has additionally been accustomed restore blurry X-ray movies of skills annexes to improve natural philosophy centralized organization trials. It aimed at the rescue of the gesticulation induced inside the talent frame or bonded possessions, and is typically used, reinstating TV movies indistinct consistently [7].

\section{RESTORATION TECHNIQUES}

Image restoration may be a technique through that a corrupted and shire image is processed in such the way that an ideal image is made [8]. Thus, restoration rebuilds those pictures whose quality is pillaged thanks to noise or command. There numerous causes for degradation like noise from the detector, camera miscues and part disturbance. There are 2 varieties of procedures are accustomed restore the image. One technique is to model the image whose quality is degraded via some reasons. Another technique referred to as image sweetening, it will increase the standard of image by applying numerous filters. Previous information of degradation is important to revive the image. The subsequent (Figure 1) showing the degradation and restoration activity. Restoration of the pictures could be achieved via 2 varieties of model particularly degradation

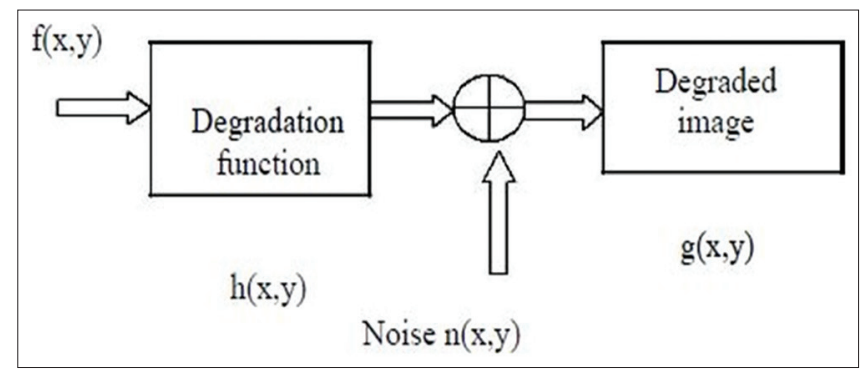

Figure 1: Degeadation Model
Model and restoration model [9]. In Figure $2 \mathrm{f}(\mathrm{x}, \mathrm{y})$ is that the unique image that is degraded by some activities. Once this on the degraded image numerous functions are applied so as to revive the image.

Below there are some methods of image restoration:

\section{Median Filter}

As the name denotes the median filter is measurements approach. During this methodology we discover the median of the picture element the replace the picture element by median of the grey levels in their neighborhood of that pixels as shown in Figure 3. The median filter is engaged to get rid of the salt and pepper noise. It's capability with significantly less blurring than liner smoothing filters of the similar size. In alternative words we will say that Median filtering could be a wide used and extremely necessary techniques of filtering and best renowned for its glorious noise reduction ability from the photographs [11].

By the filtering it keeps the perimeters whereas removing the noise. This makes the image to not blur as alternative smoothing ways. [12]

\section{Mean Filter}

\section{Arithmetic mean filters}

This category of filter is additionally known as linear filter that means all the picture element morals at intervals the window and aids in levelling the differences and blurs gift in image.

\section{Geometric mean filter}

This type is identical as mean value filter that loses less image details whereas process the image.

\section{Harmonic mean filter}

Here this type of filter is employed in things during which the information values area unit thus high, but it cannot denoise the pepper noise and is superlative for Gaussian noise and salt noise.

\section{Contra harmonic mean filter}

Here this type of filter is employed for removing salt and pepper noise however it cannot take away each of those noises at identical time. If mostly mistaken values area unit picked then it behaves as a dragon. This filter eradicates pepper noise and for a negative price it rescinds salt noise [13].

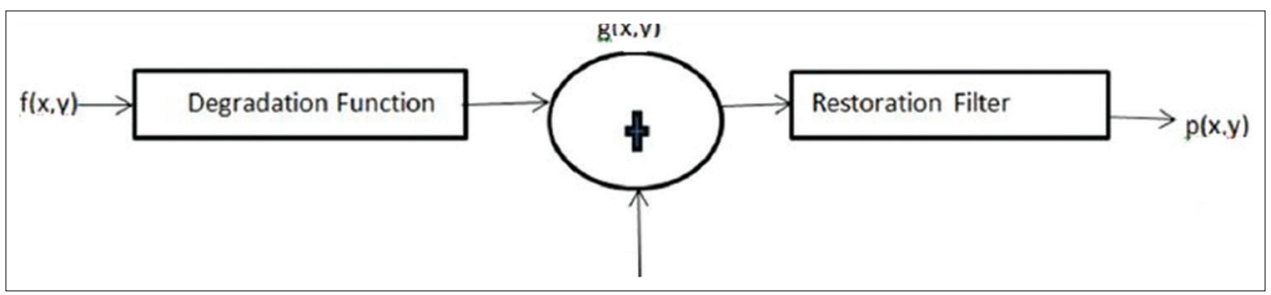

Figure 2: Degradation- restoration model [10] 


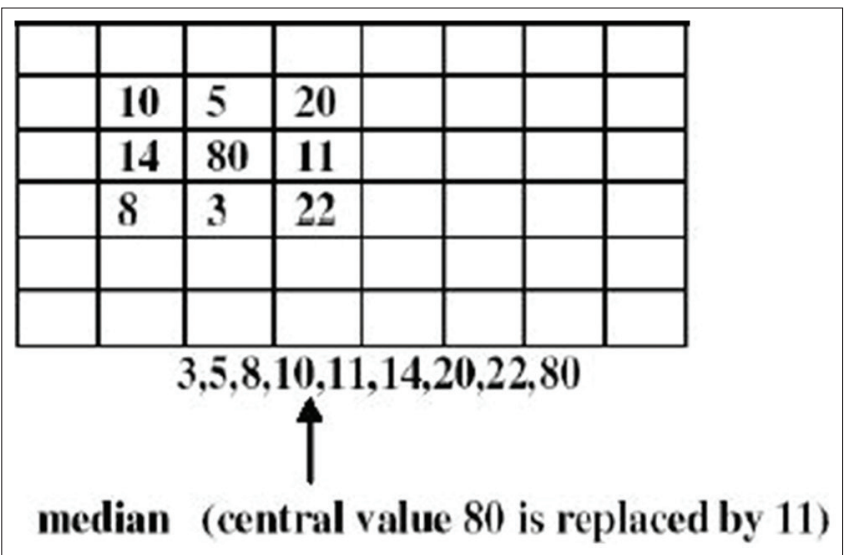

Figure 3: Median filter

\section{Adaptive Filter}

Adaptive filter is kind of linear filter that has transfer perform controlled by variable parameter. Adaptive filter use the color and grey house for removal of impulsive noise in pictures. All process is completed on the idea of color and grey house. Adaptive filter area unit won't to take away the impact of speckle noise. This may offer the simplest noise suppression results and higher preserve edges, skinny lines and image details and yield higher image quality compared to different filters [14].

\section{Weiner Filter}

This image restoration technique may be a commonplace approach that has been planned by N. Weiner, which contains each the degradation perform and applied math representative of noise into the restoration perform. It's thought of united of the simplest deblurring direct ways by that image will be reconstructed from degraded one, by mistreatment renowned foreign terrorist organization. It mechanisms with each great and low pass filter to execute deconvolution tom take away noise with density operation.

$$
\mathrm{f}=\mathrm{g} \times(\mathrm{f}+\mathrm{n})
$$

The yield image is gotten by higher than equation (2) wherever additive noise and frequency physiognomies area unit renowned [15].

\section{Neural Network Approach}

Neural networks area unit varieties of the digital computer laptop framework, with the parts of basic making ready, a high level of interconnection, versatile communication between the distinctive parts, once an element of the comes up the short neural network, it will proceed with no issue through their parallel nature [9]. factory-made Neural systems supply a powerful instrument for approximating some extent perform offer associate accumulation knowledge, yield sample moreover for the recreation perform from the category an image. A calculation, for instance, Back propagation and conjointly Perceptron habit gradient- tight ways to the harmony system strictures to the best suitable a preparation gathering of knowledge yield illustrations so tend to area unit smearing Back propagation neural network strategy for image renovation. This method is provided for the training advanced nonlinear capacities is relied upon to form the perfect structure significantly within the high repetition areas of the image and castoff a 2-layer back propagation network per the whole integration [16].

\section{Image Restoration using Wavelet Analysis}

The handicaps of Wiener Filter, normalized Filter, LucyRichardson algorithmic program, Sightless deconvolution algorithmic program LED to use rippling analysis. It needed to pre method the corrupted image previously the restoration and evaluate the degraded image to urge additional info. enforced two sorts of pre procedures. Sifting in frequency domain earlier applying rippling examination and grey equal transformation supported bar graph values. The great frequency parts typically ruled by the noise, wherever boundaries of the image area unit on the market. Thus low pass filter area unit wont to yield away the noise. Grey smooth transformation [17], escalation the distinction by spreading the grey district to deblur the image. The choosing of acceptable limit to extend distinction is incredibly necessary. Rippling examination reveals necessary particulars of the image like cutoffs and disruption points. Rippling investigation is capable to perform native analysis and to investigate a proof while not visible degradation. Solitary approach might not turn out smart consequences [18].

\section{CONCLUSIONS}

Image restoration is secondhand to increase the quality of the images that are taken from numerous resources. This review confer various image restoration methods comparable median filter, mean filter, adaptive filter, Weiner Filter, Neural Network Approach and Wavelet Analysis, wherever the images are dealt with in the time domain or in the frequency domain The supreme methods of restoration are reliant on on the application of methods in the frequency domain, Contemporaneous methods of restoration process syndicate more than one filter within and effort within a frequency domain.

\section{REFERENCES}

1. Rani S.,et al "A Brief Review on Image Restoration Techniques, International Journal of Computer Applications, Vo.150, No. 12, PP. 30-33, 2016.

2. Raval,V. and Gagnani L. " Introduction to image restoration and comparison of various methods of image restoration ", Internat ional Journal of Advanced Research in Computer Engineering \& Technology, ISSN: 2278 - 1323, Vo.1, No. 1, PP. 134-136, 2012.

3. Gunturk B. and Li X., "Image Restoration Fundamentals and Advances", CRC Press Taylor \& Francis Group “, ISN- 13: 978-1-43986956-7, 2nd printing, 2013.

4. Umbangh S. E., "Digital Image Processing and Analysis: Human and Computer Vision Applications with CVIP tools ", ISBN-13: 9781439802052, 2nd printing, Nov., 2010.

5. Birwa Raja Mohapatra, Ansuman Mishra, Sarah KumarRout. Vol.2,No.3, March 2014.A Comprehensive Review on Image 
Restoration Techniques.

6. Aizenberg I., Paliy D. and Astola, J.T. "Multilayer Neural Network based on Multi-Valued Neurons and the Blur Identification Problem", accepted to the IEEE World Congress on Computational Intelligence, Vancouver, to appear: July, 2006 Katkovnik V., Egiazarian K. and Astola J., "A spatially adaptive nonparametric image deblurring", IEEE Transactions on Image Processing, Vol. 14, No. 10,PP. 1469-1478, 2005.

7. Maurya, A.and Tiwari R. " A Novel Method of Image Restoration by using Different Types of Filtering Techniques ", International Journal of Engineering Science and Innovative Technology, ISSN: 2319-5967 ,Vol. 3, No. 4, PP $124-129,2014$.

8. Li,P.,LI,H., O., "Fuzzy techniques in image restoration research-a survey", International Journal Of Computational Cognition, Vol. 2, No. 2, PP $131-149,2004$.

9. Rani N., "Image Processing Techniques: A Review ", Today's IdeasTomorrow's Technologies, Vol. 5, No. 1, doi:10.1016,PP 40-49, 2017.

10. Maru,M., "Image Restoration Techniques: A Survey", International Journal of Computer Trends and Technology ,3(12), 2014.

11. Er. Priya Tiwari and et al." Analysis of Image Restoration Techniques for Developing Better Restoration Method "Vol.3, no. 4, .July 2013.
12. Nachtegeal, W. K.," Explore Fuzzy filters of noise reduction. The case of Gaussian noise", IEEE, 2005.

13. Nazir l., "Fuzzy techniques in image restoration research—a survey", International Journal of Computer Science and Mobile Computing, Vol. 6, No. 6, PP 405-410, 2017.

14. G.V.P.Chandra Sekhar Yadav and B. Ananda Krishna, "Study of Different Adaptive Filter Algorithms for Noise Cancellation in RealTime Environment ", International Journal of Computer Applications, Vol. 96, No. 10, PP 20-25, 2014.

15. Abd El-Fattah M. A. et al "Speech enhancement with an adaptive Wiener filter", Int J Speech Technol, Vol. 17, PP 53-64, 2014.

16. Rajput M. K. and Singh R. K., "A REVIEW ON IMAGE RESTORATION TECHNIQUES", International Journal of Advanced Technology \& Engineering Research, Vol. 5, No. 6, ISSN: 2250-3536,PP 1-7, 2015.

17. J.-S. Lee, "Digital image enhancement and noise filtering by use of local statis-tics", Pattern Analysis and Machine Intelligence. Vol.2, no. 2, pp. 165-168, Mar. 1980.

18. Kumar K. B. etal " Review on Digital Image Restoration Methods and Future Directions", International Journal of Scientific \& Engineering, Vol. 6, No. 8, ISSN 2229-5518,PP 755 - 760, 2015. 